\title{
Septic Thrombophlebitis Complicating a Peripherally Inserted Central Catheter
}

\author{
Mohammad Esmadia, c, Humera Ahsan ${ }^{\mathrm{b}}$, Dina S. Ahmad ${ }^{\mathrm{a}}$
}

\begin{abstract}
Peripherally inserted central venous catheters are associated with several complications. Thrombosis, catheter-related infection and phlebitis are well-recognized complications. However, septic thrombophlebitis is very rare. We describe a 44-year-old patient with heart failure who developed extensive septic thrombophlebitis of the right internal jugular, brachicephalic, facial veins and superior vena cava as a complication of prolonged use of a peripherally inserted central venous catheter that was inserted for dobutamine infusion. The catheter was displaced into the internal jugular vein by manipulation of the patient who used it for injection of illegal drugs. Blood cultures grew Citrobacter amalonaticus and Coagulase-negative Staphylococcus with the former growing more often making it the more likely causative agent. The patient was treated successfully with antibiotics and anticoagulation. To our knowledge Citrobacter amalonaticus has not been previously recognized to be associated with septic thrombophlebitis.
\end{abstract}

Keywords: Thrombophlebitis; Upper extremity deep vein thrombosis; Central venous catheterization; Lemierre syndrome; Citrobacter

\section{Introduction}

Peripherally inserted central venous catheters (PICCs) are

\footnotetext{
Manuscript accepted for publication February 7, 2012

${ }^{\text {a } D e p a r t m e n t ~ o f ~ I n t e r n a l ~ M e d i c i n e, ~ U n i v e r s i t y ~ o f ~ M i s s o u r i ~ S c h o o l ~ o f ~}$ Medicine, Columbia, MO, USA

${ }^{b}$ Department of Radiology, University of Missouri School of Medicine, Columbia, MO, USA

${ }^{\mathrm{c} C}$ Corresponding author: Mohammad Esmadi, One Hospital Dr., DC043.00 Health Sciences Center, Columbia, MO 65212, USA. Email: mohsmadi@hotmail.com
}

doi:10.4021/jmc560w currently being more widely used with high efficacy for outpatient administration of several medications and parentral nutrition. Complications of PICCs include occlusion, phlebitis, catheter-related infection, catheter malposition, catheter fracture, venous thrombosis and very rarely septic thrombophlebitis of central deep veins. PICCs have reduced rates of immediate traumatic complications like pneumothorax, hemothorax and arterial injury compared to centrally insert venous catheters (CVCs). However, PICCs have higher rates of malfunction and phlebitis on the long-term [1].

Septic thrombophlebitis of the internal jugular vein has been classically associated with oropharyngeal infections [2] and very rarely with CVCs [3-7] and PICCs [8-10].

\section{Case Report}

A 44-year-old patient known to have alcohol-induced cardiomyopathy with ejection fraction of $20-25 \%$ was started on dobutamine infusion via a PICC. The PICC was inserted in an inpatient setting and correct positioning was confirmed by a portable chest x-ray (Fig. 1A). The patient was discharged with weekly follow ups in the cardiology clinic weekly. Two months later, the PICC was noted in the clinic to have resistance for blood drawing. The patient was given an appointment for removal of the PICC and insertion of another one. However, he did not adhere to the appointment and lost follow up. One month later, the patient presented to the emergency room with fever, chills, headache, neck pain and right shoulder pain. On physical examination, tenderness and crepitus were noticed in the right side of the neck. His temperature was $38.9^{\circ} \mathrm{C}$, heart rate 112 beats per minute and blood pressure $97 / 69$. His WBC count was $23.910^{-3} / \mu \mathrm{L}$, CRP $19.9 \mathrm{mg} / \mathrm{dL}$ and ESR $62 \mathrm{~mm} / \mathrm{hr}$. Chest x-ray showed displacement of the PICC into the right internal jugular vein (Fig. 1B). The catheter was immediately removed in the emergency room. CT scan of the neck and chest showed admixture of air and fluid within the superior vena cava, right brachiocephalic, internal jugular and facial veins consistent with septic thrombophlebitis (Fig. 2A, B, C). Piperacillin/ Tazaobactam and Vancomycin were started empirically along with Heparin. 


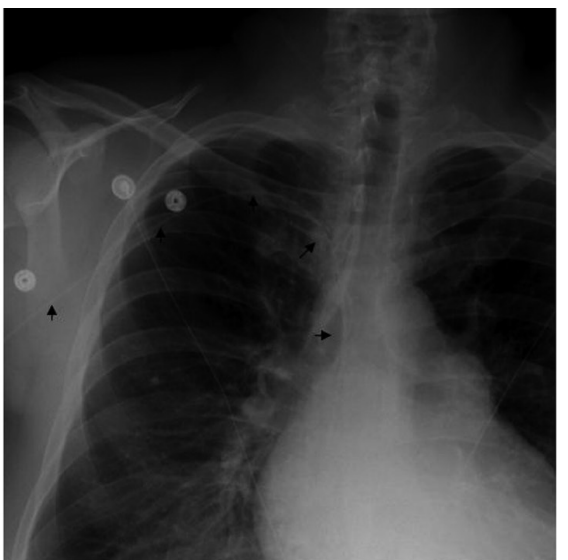

A

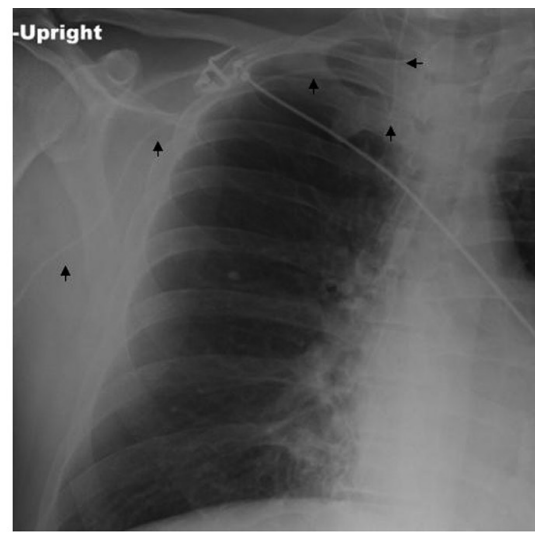

B

Figure 1. A, Portable chest x-ray after placing the PICC showing a correct positioning (arrows). B, Portable chest x-ray 3 months later showing displacement of the PICC into the right internal jugular vein (arrows).

Over the course of the first week of hospitalization, the patient remained to have low-grade fever with positive blood cultures for both Citrobacter amalonaticus and Coagulase-negative Staphylococcus (CONS) with Citrobacter amalonaticus growing more often making it the more likely causative agent of the thrombophlebitis. Citrobacter amalonaticus had only intermediate sensitivity to Piperacillin/ Tazaobactam, so it was replaced with Mropenem on day 6. On day 7, CT scan of the neck was repeated and it showed near complete thrombosis of the superior vena cava, right brachicephalic, subclavian, internal jugular and facial veins (Fig. 3A, B). The patient's condition overall improved after the first week and blood cultures started to sterilize. On day 13, Intravenous (IV) antibiotics were stopped and he was started on oral sulfamethoxazole-trimethoprim to which the Citrobacter amalonaticus was sensitive.

The patient admitted using illegal IV drugs through the
PICC. He manipulated it several times which explains the malpositioning of the line into the right internal jugular vein. He was discharged home on sulfamethoxazole-trimethoprim and warfarin after three weeks of hospitalization. Warfarin was stopped 3 months later in the clinic.

\section{Discussion}

PICCs have been recently used with great efficacy. The rate of complications of PICCs ranges from 35-65\% [1, 11-12]. In some studies, PICCs had lower rates of catheter-related bloodstream infections in the outpatient setting compared to CVCs [12-13], while PICCs and CVCs had similar rates in the inpatient setting [14]. The rate of thrombosis varies from $0.3 \%$ to $56 \%$. Low rates have been reported in retrospective studies which only diagnosed symptomatic thrombosis,

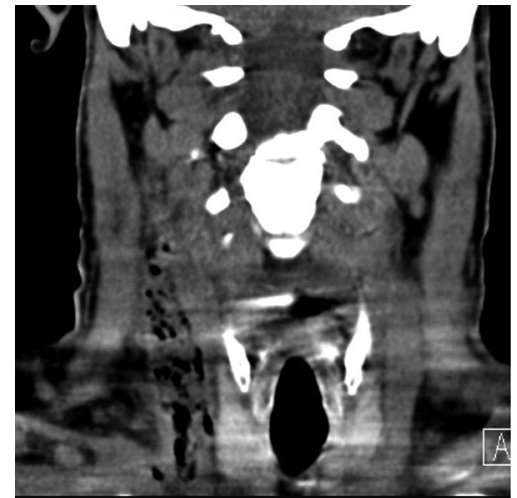

A

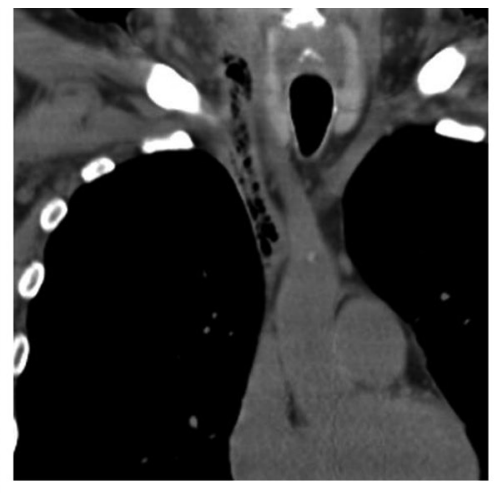

B

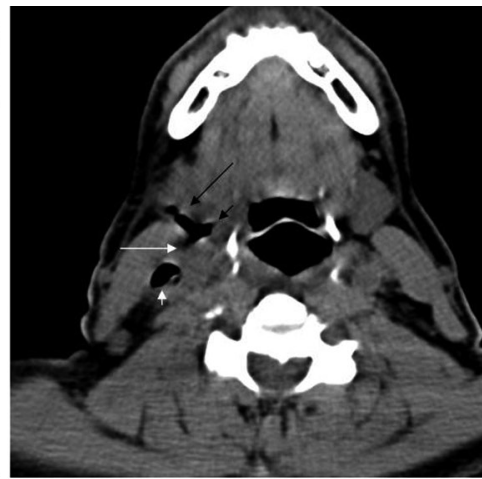

$\mathrm{C}$

Figure 2. A, Non-contrast CT scan of the neck showing admixture of air and fluid in the right internal jugular vein. B, Non-contrast CT scan of the chest showing extension of the thrombophlebitis to the right brachicephalic vein and superior vena cava. C, Non-contrast CT scan of the neck showing air in the internal jugular vein (short white arrow), common facial vein (long white arrow), external facial vein (long black arrow) and internal facial vein (short black arrow). 

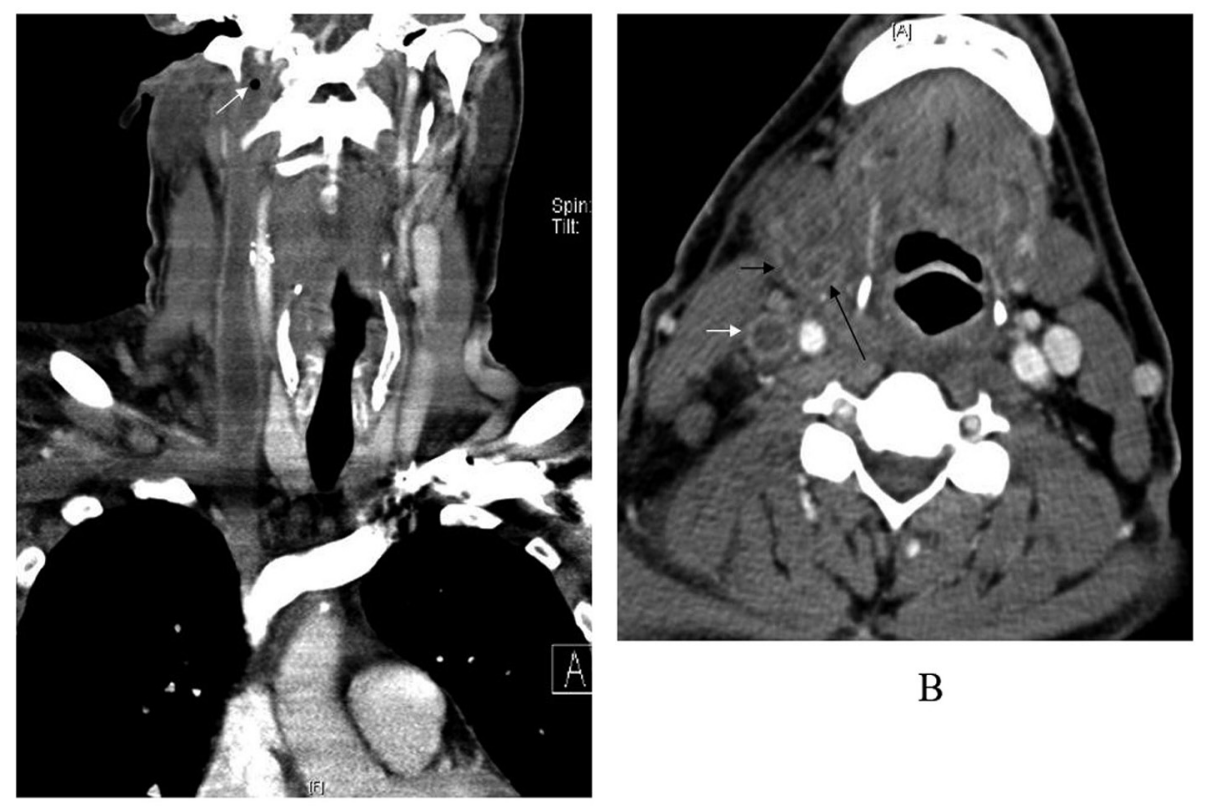

B

A

Figure 3. A, CT scan of the neck and chest with contrast showing thrombosis of the right internal jugular vein, right subclavian vein, right brachiocephalic and superior vena cava with a small residual pocket of air inferior to the jugular foramen (arrow). B, CT scan of the neck with contrast showing thrombosis of the internal jugular vein (white arrow), external facial vein (short black arrow) and internal facial vein (long black arrow).

while prospective studies have reported higher rates [15].

Septic thrombophlebitis of the internal jugular vein has been classically associated with oropharyngeal infections as part of Lemierre syndrome which sometimes is associated with septic emboli to the lungs [16]. Very few cases of septic thrombophlebitis of the superior vena cava, subclavian vein and internal jugular vein due to central venous catheters [37] and PICCs [8-10] have been reported previously.

Our case demonstrates extensive septic thrombophlebitis of the deep veins as a very rare but serious complication of PICCs. The development of septic thrombophlebitis was related to prolonged use and manipulation by the patient during injection of illegal IV drugs. Septic thrombophlebitis complicating PICCs is a medical emergency. Early recognition, removal of the PICC, and administration of IV antibiotics and anticoagulation are essential for successful management. CT scan is a useful modality for tracking the extent of the thrombophlebitis. To our knowledge, Citrobacter amalonaticus has not been described previously to be associated with thrombophlebitis [16].

\section{Acknowledgment}

Dr Ashraf Al-Dadah was contributed to the details of the clinical course of the patient. Work was done at University of Missouri School of Medicine, Columbia, MO.

\section{Conflict of Interest}

No.

\section{Funding}

No.

\section{References}

1. Smith JR, Friedell ML, Cheatham ML, Martin SP, Cohen MJ, Horowitz JD. Peripherally inserted central catheters revisited. Am J Surg. 1998;176(2):208-211.

2. Sinave CP, Hardy GJ, Fardy PW. The Lemierre syndrome: suppurative thrombophlebitis of the internal jugular vein secondary to oropharyngeal infection. Medicine (Baltimore). 1989;68(2):85-94.

3. Verghese A, Widrich WC, Arbeit RD. Central venous septic thrombophlebitis--the role of medical therapy. Medicine (Baltimore). 1985;64(6):394-400.

4. Kaufman J, Demas C, Stark K, Flancbaum L. Catheterrelated septic central venous thrombosis--current therapeutic options. West J Med. 1986;145(2):200-203.

5. Richardson T, Mehler PS. Persistent staphylococcal bacteremia, lest we forget the old catheter site. South Med J. 
2003;96(1):112-113.

6. Hennigan A, Collinge N, Halker R, Agraharkar M. Cuffed-catheter-related Lemierre syndrome. Hemodial Int. 2004;8(4):400-403.

7. Terada Y, Mitsui T, Jikuya T, Kaminishi Y, Watanabe K. Infected thrombophlebitis of the right internal jugular vein. J Vasc Surg. 1996;24(6):1066-1067.

8. Giuffrida DJ, Bryan-Brown CW, Lumb PD, Kwun KB, Rhoades HM. Central vs peripheral venous catheters in critically ill patients. Chest. 1986;90(6):806-809.

9. Merrell SW, Peatross BG, Grossman MD, Sullivan JJ, Harker WG. Peripherally inserted central venous catheters. Low-risk alternatives for ongoing venous access. West J Med. 1994;160(1):25-30.

10. Andes DR, Urban AW, Acher CW, Maki DG. Septic thrombosis of the basilic, axillary, and subclavian veins caused by a peripherally inserted central venous catheter. Am J Med. 1998;105(5):446-450.

11. Cowl CT, Weinstock JV, Al-Jurf A, Ephgrave K, Murray JA, Dillon K. Complications and cost associated with parenteral nutrition delivered to hospitalized patients through either subclavian or peripherally-inserted cen- tral catheters. Clin Nutr. 2000;19(4):237-243.

12. Chlebicki MP, Teo EK. Review of peripherally inserted central catheters in the Singapore acute-care hospital. Singapore Med J. 2003;44(10):531-535.

13. Maki DG, Kluger DM, Crnich CJ. The risk of bloodstream infection in adults with different intravascular devices: a systematic review of 200 published prospective studies. Mayo Clin Proc. 2006;81(9):11591171.

14. Safdar N, Maki DG. Risk of catheter-related bloodstream infection with peripherally inserted central venous catheters used in hospitalized patients. Chest. 2005;128(2):489-495.

15. Chemaly RF, de Parres JB, Rehm SJ, Adal KA, Lisgaris MV, Katz-Scott DS, Curtas S, et al. Venous thrombosis associated with peripherally inserted central catheters: a retrospective analysis of the Cleveland Clinic experience. Clin Infect Dis. 2002;34(9):1179-1183.

16. Chirinos JA, Lichtstein DM, Garcia J, Tamariz LJ. The evolution of Lemierre syndrome: report of 2 cases and review of the literature. Medicine (Baltimore). 2002;81(6):458-465. 